
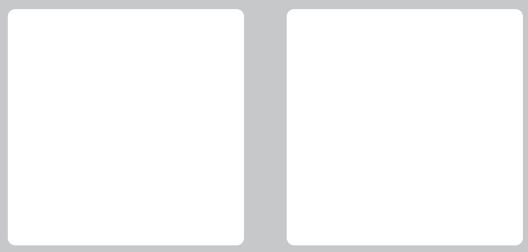

\title{
EFEITOS DA AGILIDADE ORGANIZACIONAL NO COMPROMETIMENTO ORGANIZACIONAL: O PAPEL MEDIADOR DO EMPOWERMENT PSICOLÓGICO
}

\section{Effects of Organizational Agility on Organizational Commitment: The Mediating Role of Psychological Empowerment}

\author{
Januário José Monteiro \\ Universidade Federal de Santa Catarina (UFSC). \\ email:januariomonteiromonteiro@gmail.com
}

Edicreia Andrade dos Santos

Universidade Federal do Paraná (UFPR).

email:edicreiaandrade@yahoo.com.br

\author{
Rogério João Lunkes \\ Universidade Federal de Santa Catarina (UFSC). \\ email: rogeriolunkes@hotmail.com
}

\section{Darci Schnorrenberger}

Universidade Federal de Santa Catarina (UFSC).

email:darcisc@gmail.com

\section{RESUMO}

Este estudo tem como objetivo examinar o efeito da agilidade organizacional no comprometimento organizacional mediado pelo empowerment psicológico. A pesquisa foi desenvolvida a partir de um questionário que levantou a percepção de 87 funcionários de um Hospital com atendimento de excelência a emergências cardiológicas de Florianópolis (SC). Foram testadas três hipóteses e os dados foram analisados a partir da modelagem de Equações Estruturais no PLS. Dentre os achados confirmou-se que há relação positiva significante entre a agilidade e o comprometimento organizacional, além de que o empowerment psicológico está diretamente relacionado com o comprometimento organizacional, e também medeia positivamente a relação entre a agilidade organizacional e o comprometimento. Infere-se que os funcionários percebem que sua organização é ágil, quando essa adota políticas que a permitem adaptar-se às mudanças no ambiente organizacional. Essa percepção aumenta o empowerment psicológico dos funcionários e consequentemente afeta o sentimento de apego com a organização.

Palavras-Chave: Agilidade organizacional; Comprometimento organizacional; Empowerment psicológico; Hospital

\section{ABSTRACT}

This study aims to examine the effect of organizational agility on organizational commitment mediated by psychological empowerment. The research was developed based on a questionnaire that raised the perception of 87 employees of a Hospital of excellence attendance at cardiological emergencies in Florianopolis (SC). Three hypotheses were tested and the data were analyzed using the Structural Equations technique in PLS. Among the results obtained, it was confirmed that there is a positive and significant relationship between organizational agility and organizational commitment, besides that psychological empowerment is directly related to organizational commitment and positively mediates the relationship between organizational agility and commitment. It is inferred that employees perceive that their organization is agile when it adopts policies that allow it to adapt to the changes in the organizational environment. This perception increases the psychological empowerment of employees and consequently affects their sense of attachment to the organization.

Key-words: Organizational agility; Organizational commitment; Psychological Empowerment; Hospital 


\section{INTRODUÇÃO}

A literatura acerca de agilidade organizacional é extensa e inicialmente foi estudada no setor industrial (NAFEI, 2016). Além de versar acerca da capacidade da empresa em responder tempestivamente às mudanças, também foca na flexibilidade, inovação, qualidade dos produtos e/ou serviços e lucratividade, no intuito de alcançar melhores resultados e responder às necessidades dos clientes (YUSUF; SRHADI; GUNASEKARAN, 1999; NAFEI, 2016).

Estudos anteriores que investigaram a agilidade organizacional constataram que especialmente em ambiente de mudanças é necessário que a organização tenha habilidades para adaptar-se às estratégias, processos e recursos no intuito de responder às constantes volatilidades ambientais (ALMAHAMID; AWWAD; MCADAMS, 2010). A agilidade é composta pelas dimensões de responsabilidade, competência, flexibilidade e rapidez (MEHDIBEIGI; DEHGHANI; YAGHOUBI, 2016), que já foram testadas em alguns estudos. Por exemplo, Porkiani e Hejinipoor (2013) e Bahrami et al. (2016) a relacionaram com a inteligência e aprendizagem organizacional. Além dessas variáveis também evidenciou-se relações com o desempenho, estilo de liderança e empowerment psicológico (AVOLIO et al., 2004; PORKIANI; HEJINIPOOR, 2013; BAHRAMI et al., 2016; MEHDIBEIGI; DEHGHANI; YAGHOUBI, 2016). Em relação ao campo de pesquisa nos quais os estudos foram desenvolvidos destacam-se os tribunais (PORKIANI; HEJINIPOOR, 2013); esportivo (MIRZAZADEH; SAFFAR, 2014); bancário (CHAMANIFARD, 2015) e hospitalar (BAHRAMI et al., 2016). Este estudo explora a temática de agilidade organizacional na área hospitalar.

Outra variável que pode ser relacionada com a agilidade organizacional é o comprometimento organizacional, entendido como a força que impulsiona o indivíduo a se identificar com a organização, por meio dos seus valores e objetivos (STEERS, 1977; BAHRAMI et al., 2016). A literatura a cerca do tema é extensa e seu conceito tem sido associado a variáveis individuais e organizacionais (MATHIEU; ZAJAC, 1990; SPANUTH; WALD, 2017). Por exemplo, Bahrami et al. (2015) investigaram o comprometimento de 90 enfermeiros em dois hospitais em Yazd (Irã), e concluíram que o comprometimento destes profissionais com o hospital era satisfatório à medida em que a instituição proporcionava boas condições de trabalho. Uma constatação importante da pesquisa foi de que quanto melhor for o clima organizacional, maior é o comprometimento organizacional.

Conger e Kamungo (1988) apontam que um dos fatores que impulsiona o comprometimento organizacional é o empoderamento do indivíduo, o qual atua como motivador intrínseco e que influencia na satisfação do indivíduo. O empowerment psicológico é definido como o aumento da motivação intrínseca movida por cognições por meio das quais, os indivíduos se responsabilizam pelas estratégias e resultados da empresa (WELLINS; BYHAM; WILSON, 1991; SPREITZER, 1995).

Neste estudo, o empowerment psicológico é testado como variável mediadora entre a agilidade e o comprometimento organizacional. Essa proposta está teoricamente alinhada aos estudos de Avolio et al. (2004) e Farahani e Salimi (2015), que usaram o empowerment psicológico como mediador afetando o comprometimento organizacional. Ademais, esta pesquisa segue as recomendações de Conway e Monks, (2009) e Bin Jomah (2017), que afirmaram serem necessárias mais pesquisas que relacionem outras variáveis ao comprometimento organizacional. Como por exemplo, examinar a agilidade organizacional a partir de diferentes dimensões e relacioná-la ao comprometimento, e ao mesmo tempo observar de que maneira o empowerment psicológico exerce influência nessa relação.

Diante do exposto, propõe-se a seguinte questão de pesquisa: qual o efeito da agilidade organizacional no comprometimento organizacional mediado pelo empowerment psicológico? Para tanto, o objetivo deste estudo consiste em examinar o efeito da agilidade organizacional no comprometimento organizacional, mediado pelo empowerment psicológico de funcionários de diversas funções de um hospital localizado na cidade de Florianópolis/SC.

Este estudo contribui para que as organizações hospitalares alcancem maior comprometimento dos seus profissionais, o que pode levar ao melhor desempenho, pois como afirmam Vandenberghe e Tremplay (2008), o comprometimento organizacional exerce um impacto positivo no desempenho. A me- 
dida que a organização se torna mais ágil maior é o empowerment psicológico dos profissionais, por isso os funcionários se comprometem cada vez mais com a organização, fator que facilita a coesão da equipe de trabalho, aumenta a criatividade e o engajamento dos profissionais, tornando a organização mais competitiva no mercado (MATHIEU; ZAJAC, 1990).

As organizações ágeis estabelecem vantagem competitiva por estarem entre as primeiras a identificar ameaças e oportunidades em ambientes de negócios em constante mudança, e por serem mais hábeis do que os concorrentes atuais e potenciais em explorar as oportunidades repetidamente ao longo do tempo (NAFEI, 2016). Por isso, em nível prático este estudo busca contribuir para que as organizações hospitalares e outras similares possam alcançar melhor desempenho, ao se tornarem mais ágeis e com maior comprometimento por parte de seus profissionais. Quanto à contribuição social, argumenta-se que processos relacionados ao comprometimento organizacional têm implicações na sociedade como um todo, tendo em vista que a satisfação é preditora do comprometimento, logo se a organização permite a criação de um ambiente mais favorável aos interesses dos profissionais, isso levará a uma prestação de serviço de melhor qualidade, o que possibilitará à sociedade em geral se beneficiar dessa melhoria (MOWDAY; PORTER; STEERS, 2013).

\section{REFERENCIAL TEÓRICO}

\subsection{Agilidade Organizacional}

A literatura organizacional indica que o conceito de agilidade emergiu primariamente como um novo paradigma de fabricação, proposto como uma estratégia para permitir que as empresas manufatureiras mantivessem sua competitividade no século XXI. Por isso, grande parte da ênfase da temática é dada no processo e na tecnologia no contexto da manufatura ágil, ou em amplas questões organizacionais no contexto de um ambiente de manufatura (NAFEI, 2016). No entanto, o conceito de agilidade tem apelo universal e aplicabilidade a todas as organizações afetadas pela realidade ambiental dinâmica e imprevisível, e não apenas pela manufatura.
Assim, a agilidade organizacional é entendida sob diferentes perspectivas e é definida como um dos fatores de sucesso para organizações em ambientes de alta concorrência (SHARIFI; ZHANG, 2000; CHARBONNIER-VOIRIN, 2011; NAFEI, 2016). Ela é a aplicação bem-sucedida de bases competitivas como, competência, responsabilidade, rapidez/velocidade, flexibilidade, inovação e qualidade, por meio da integração de recursos reconfiguráveis e melhores práticas para fornecer produtos e serviços orientados para o cliente em um ambiente em rápida mudança (MEHDIBEIGI; DEHGHANI; YAGHOUBI, 2016; NAFEI, 2016).

A agilidade é apontada como imprescindível para a sobrevivência das empresas, dado que o ambiente organizacional está cada vez mais competitivo (CHARBONNIER-VOIRIN, 2011; NAFEI, 2016). A velocidade e a flexibilidade considerados como os principais atributos de uma organização ágil, oferecem resposta eficaz à mudança e à incerteza; respostas proativas às mudanças em ambientes turbulentos e capacidade de sobreviver e progredir no ambiente variável e imprevisível (NAFEI, 2016). Portanto, a agilidade organizacional se concentra nas habilidades da organização em se adaptar às estratégias, processos e recursos, tendo por finalidade responder às mudanças no ambiente (ALMAHAMID; AWWAD; MCADMAS, 2010).

Diversos estudos empíricos testaram a agilidade organizacional como proxy de entrada em diversos modelos, e observaram que ela reflete em melhores resultados da organização (SAMBAMURTHY; BHARADWAJ; GROVER, 2003), e na criação de vantagem competitiva (BROWN; BESSANT, 2003). A imprecisão dos construtos teóricos desenvolvidos para a mensuração da agilidade organizacional justifica a razão pela qual, há na literatura distintos olhares sobre a temática (ex.: CHAMANIFARD,2015; BAHRAMI et al.,2016; HHAMAD; VOZGAT, 2017).

Chamanifard (2015) investigou o impacto das dimensões da agilidade organizacional no comprometimento e os resultados destacaram que a responsabilidade, competência, flexibilidade e rapidez estão positivamente relacionadas com o comprometimento organizacional. Bahrami et al. (2016) examinaram a relação entre a inteligência organizacional e agilidade organizacional, tendo como variável mediadora 
a aprendizagem organizacional. O estudo classificado como analítico e transversal foi realizado em 4 hospitais, e envolveu 370 funcionários desde administrativos até médicos. Os resultados evidenciaram uma relação significativa entre a inteligência organizacional e a agilidade organizacional. Por fim, Hhamad e Vozgat (2017) testaram a relação entre a agilidade organizacional e a aprendizagem organizacional e constataram uma relação significativa, o que demonstra que os gestores de organizações ágeis são mais propensos à aprendizagem.

Observa-se que a agilidade organizacional foi testada em diversos ambientes, tanto como variável dependente quanto independente. Foi ainda relacionada com outras variáveis como inteligência organizacional, aprendizagem organizacional, empowerment psicológico, desempenho organizacional e comprometimento organizacional (PORKIANI; HEJINIPOOR, 2013; MIRZAZADEH; SAFFAR, 2014; BAHRAMI et al., 2016; MEHDIBEIGI; DEHGHANI; YAGHOUBI, 2016).

\subsection{Comprometimento Organizacional}

O comprometimento organizacional é a força que impulsiona o indivíduo a se identificar com a organização (STEERS, 1977). Em outras palavras, ele resulta das relações afetivas do indivíduo em relação as características de sua organização empregadora, na qual a preocupação inicial consiste no sentimento de apego dos funcionários aos objetivos, visão e missão da organização, gerando resultados positivos como bem-estar no trabalho (ALLEN; MEYER, 1990).

O comprometimento organizacional pode ser observado sob duas abordagens, sendo o enfoque no apego do indivíduo com a organização e a intenção de continuar no emprego (KIM et al., 2012). Essas duas abordagens podem ser analisadas sob o olhar de três componentes: afetivo, dimensão de continuação e normativo (ALLEN; MEYER, 1990). O comprometimento afetivo refere-se às percepções dos funcionários sobre seu apego emocional à organização e aos objetivos, e os fatores individuais (por exemplo, personalidade, orientação a valores, educação e idade), e fatores organizacionais (por exemplo, acreditar que os papéis e objetivos do trabalho são claramente definidos e receber apoio gerencial), que podem influenciar o nível de comprometimento afetivo. A dimensão de continuação do comprometimento representa um vínculo cognitivo entre os funcionários e sua organização, e baseia-se no pressuposto de que os indivíduos não abandonam uma organização se perderem seus benefícios ou terem redução salarial, pois incorrerão em despesas de procura de emprego e correrão o risco de ficarem desempregados. O comprometimento normativo refere-se a sentimentos típicos de obrigação de permanecer na organização, e baseia-se em uma ideologia ou em um senso de obrigação. Assim, o funcionário se sente obrigado a permanecer na organização porque é a decisão moral e certa a ser realizada (ALLEN; MEYER, 1990).

O comprometimento organizacional é importante no mais diferentes tipos de organizações. Thanacoody, Newman e Fuchs (2014) destacam a importância do comprometimento organizacional nas organizações hospitalares, afirmando que em um ambiente caracterizado por constantes mudanças e incertezas institucionais, formuladores de políticas e acadêmicos começaram a reconhecer que uma força de trabalho engajada, saudável e motivada é vital para a prestação de cuidados de saúde de alta qualidade. Assim, conclui-se que o comprometimento dos funcionários deve ser obtido pela organização e não assumido por ela, o que implica que as organizações devem tentar ser proativas em influenciar o nível de comprometimento organizacional em seus funcionários.

Baird, Tung e Yu (2019) argumentam que os funcionários se engajam no comprometimento com a organização em resposta a alterações do ambiente, portanto, o conhecimento ao respeito do impacto das mudanças no comprometimento dos funcionários é crucial. Os autores argumentam também que o desempenho dos hospitais, suas instalações adequadas (instalações médicas, instalações de apoio e recursos da equipe) influencia o nível de comprometimento, que por sua vez reflete no desempenho do hospital no que tange ao atendimento ao paciente e à eficácia operacional.

O comprometimento dos funcionários, também decorre da agilidade da organização, ou seja, são respostas às reações e adaptações às mudanças (ALAVI et al., 2014). A literatura, embora ainda limitada apresentou indícios de associação entre a agilidade 
organizacional e o comprometimento organizacional. Por exemplo, Chamanifard (2015) apontou que características como responsabilidade, competência, flexibilidade e rapidez inerente à organização aumentam o comprometimento organizacional. Portanto, conjectura-se nesta pesquisa que a agilidade com que a organização responde as suas mudanças também influencia no comprometimento do funcionário. Pois, quanto mais ágil for a organização maior será o sentimento de apego, o que gera o comprometimento por parte do indivíduo. Com isso apresenta-se a primeira hipótese

$\mathrm{H}_{1}$. Há relação positiva entre a agilidade organizacional e o comprometimento organizacional.

\subsection{Empowerment Psicológico}

O empowerment é um dos principais fatores de sucesso da organização, definido como o processo interno do indivíduo ou externo de se sentir empoderado (THOMAS; VELTHOUSE, 1990; SHAPIRA-LISHCHINSKY; TSEMACH, 2014). O empowerment psicológico é definido como o aumento da motivação intrínseca movida por cognições, mediante as quais o funcionário aplica no desempenho de suas funções (SPREITZER, 1995). Em linhas gerais, o empowerment psicológico é como um processo de percepção dos indivíduos sobre sua própria eficácia em comparação com os outros membros da organização, juntamente com ajuda de procedimentos e técnicas formais e informais para incentivar a sua efetividade (THOMAS; VELTHOUSE, 1990; SHAPIRA-LISHCHINSKY; TSEMACH, 2014).

As cognições do empowerment psicológico, de acordo com Thomas e Velthouse (1990), são agrupadas em quatro grupos, sendo: (i) competência que refere-se à capacidade do indivíduo em realizar determinada tarefa com sucesso; (ii) impacto, que diz respeito a diferença que o trabalho do funcionário pode fazer no alcance dos objetivos organizacionais e na forma como ele acredita na sua influência sobre os resultados; (iii) significado que refere-se as crenças e valores do indivíduo relacionado ao exercício das suas funções, e; (iv) autodeterminação que remete a forma como o funcionário toma decisões, atrelado a sua autonomia no decurso de suas funções. O empowerment psicológico, portanto, consiste em todas as quatro dimensões, pois profissionais empoderados não aguardam passivamente as instruções, mas são ativos e afetam seu ambiente de trabalho, levando a uma maior eficiência. Os profissionais capacitados acreditam que são importantes e influentes na organização, e sentem maior senso de comprometimento.

O conceito de empowerment psicológico desempenha um papel importante em construções comportamentais, emocionais e cognitivas (WANG, 2015), tal como satisfação no trabalho, comportamento de cidadania organizacional, comprometimento organizacional etc. O comprometimento organizacional, por exemplo, é percebido como um compromisso de afetividade, normativo ou de continuiação (ALLEN; MEYER, 1990). O empoderamento psicológico visa aumentar os níveis de comprometimento nas organizações (BIN JOMAH, 2017). Assim, de acordo com Bin Jomah (2017), o empowerment psicológico pode evocar o comprometimento organizacional, uma vez que o impacto permite que os funcionários participem da modelagem do sistema de uma organização à qual estão conectados. A autodeterminação fornece aos trabalhadores uma voz nos processos de decisão relacionados ao trabalho e maior controle sobre ele, consequentemente, levando ao aumento do envolvimento nas operações da organização. Os sentidos de competência permitem que os indivíduos acreditem que são capazes de desempenhar suas funções com sucesso e habilidades e, por sua vez, incentivá-los a envidar esforços consideráveis enquanto trabalham na organização. Para que um trabalho seja considerado significativo, ele deve fornecer uma linha adequada entre os fundamentos e finalidades das funções organizacionais de uma pessoa e seus atributos de valor pessoal (BIN JOMAH, 2017). Considerando estas dimensões do empowerment, conjectura-se que ele afete o comprometimento organizacional. Para explorar essa relação, propõe-se testar a seguinte hipótese.

$\mathrm{H}_{2}$. Há relação positiva entre o empowerment psicológico e o comprometimento organizacional.

O empowerment psicológico também pode ser um mediador entre variáveis organizacionais e individuais (AVOLIO et al., 2004; FARAHANI; SALIMI, 
2015). Avolio et al. (2004) testaram o empowerment psicológico como variável mediadora entre os efeitos da liderança transformacional e o comprometimento organizacional, junto a 520 médicos e enfermeiros de um hospital de Cingapura. Os achados mostraram que o empowerment funciona como variável mediadora exercendo influência positiva no comprometimento organizacional. Farahani e Salimi (2015) examinaram a relação entre o empowerment psicológico dos funcionários e a agilidade organizacional. O estudo foi desenvolvido em uma indústria no Irã e teve como amostra 329 indivíduos. Os resultados evidenciaram que há uma relação positiva entre agilidade organizacional e o empowerment psicológico.

Argumenta-se que a agilidade organizacional, pelas suas características de rapidez, flexibilização (SHARIFI; ZHANG, 1999), pode potencializar o comprometimento dos indivíduos na organização (BIN JOMAH, 2017). Essa relação pode ser reforçada à medida em que os indivíduos alcançam alto grau de empowerment psicológico. Assim, conjectura-se a seguinte hipótese de pesquisa:

$\mathrm{H}_{3}$. O empowerment psicológico atua como mediador no aumento do comprometimento organizacional por meio da agilidade organizacional.

Face ao contexto apresentado, evidencia-se o modelo de pesquisa, conforme Figura 1.

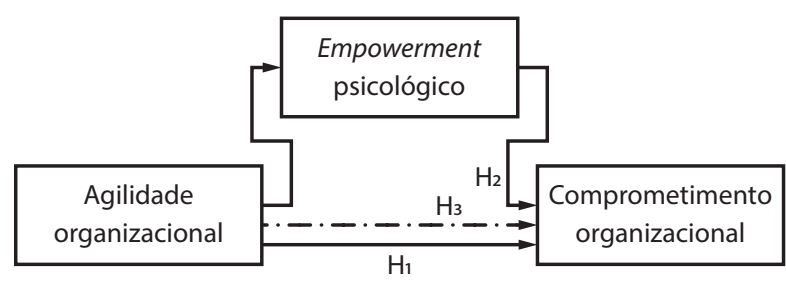

Figura 1 Desenho da pesquisa

A pesquisa fundamenta-se na literatura sobre agilidade organizacional (SHARIFI; ZHANG,1999), empowerment psicológico (SPREITZER,1995), e comprometimento organizacional (ALLEN; MEYER,1990). Observa-se que o empowerment psicológico funciona como variável mediadora da relação entre a agilidade organizacional e o comprometimento. Argumenta-se que quando a empresa se torna mais ágil, mais liberdade é dada aos funcionários, que contribui no seu empowerment psicológico, e afeta seu comprometimento com a organização.

\section{PROCEDIMENTOS METODOLÓGICOS}

\subsection{População e Amostra}

Esta pesquisa é de caráter descritivo, quantitativo e foi realizada por meio de uma survey, junto a funcionários de distintas áreas funcionais de um hospital situado na cidade de Florianópolis/SC. O contato com os funcionários ocorreu primeiramente por intermédio do setor de Recursos humanos, que viabilizou a aplicação dos questionários de forma presencial, durante o período de 20 de dezembro de 2018 a 15 de janeiro de 2019.

O tamanho mínimo da amostra necessária foi calculado no software $G^{\star}$ Power (FAUL; ERDEFELDER; LANG, 2009). Dado que o modelo apresenta duas variáveis preditoras (agilidade organizacional e empowerment psicológico) e calcula seus efeitos totais sobre a variável dependente (Comprometimento organizacional), com nível de significância de 5\% que proporciona um poder explicativo de $95 \%$, tem-se um mínimo de 78 respostas válidas. Desse modo a amostra final foi de 87 respostas válidas que atendeu aos critérios.

\subsection{Instrumento de Pesquisa e Coleta e Análise dos Dados}

$\mathrm{O}$ instrumento de pesquisa baseou-se na literatura de agilidade organizacional (SHARIFI; ZHANG, 1999) empowerment psicológico (SPREITZER, 1995), e comprometimento organizacional (ALLEN; MEYER, 1990). Cuidados adicionais foram tomados, como procedimentos de back-translation (BRISLIN, 1970), e pré-testes com dois professores doutores que atuam na área de administração e contabilidade e três profissionais da saúde. Esse processo permitiu a readequação na redação das questões, bem como adequação ao ambiente de pesquisa. Em alguns casos, incluiu-se nas assertivas o contexto organizacional em estudo, de modos a deixá-las mais claras para os respondentes (Ex. Nosso hospital tem a capacidade de desafiar e superar as novas entradas de concorrentes no mercado).

O questionário foi formado por 40 questões agrupadas em três construtos que foram mensuradas 
Tabela 1 Construtos e assertivas do instrumento da pesquisa.

\begin{tabular}{|c|c|c|}
\hline Construto & Variáveis & Assertivas \\
\hline \multirow{19}{*}{ 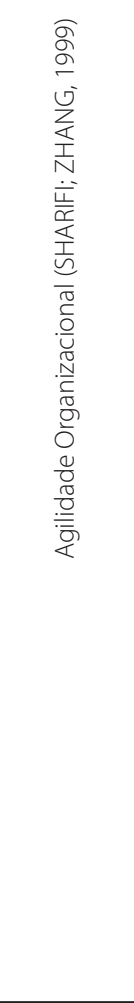 } & \multirow[t]{5}{*}{ Responsabilidade } & Nosso hospital toma decisões rápidas sobre a reação à mudança de preço do mercado. \\
\hline & & Nosso hospital tem a tendência de perceber mudanças nas necessidades dos clientes/pacientes. \\
\hline & & Nosso hospital geralmente realiza reuniões interdepartamentais regulares sobre reações a mudanças externas. \\
\hline & & As atividades interdepartamentais são bem coordenadas em nosso hospital. \\
\hline & & Os elogios do cliente são percebidos em nosso hospital. \\
\hline & \multirow[t]{5}{*}{ Competência } & Nosso hospital consegue implementar os planos no prazo. \\
\hline & & Nosso hospital tem a capacidade de desafiar e superar as novas entradas de concorrentes no mercado. \\
\hline & & $\begin{array}{l}\text { Nosso hospital tem a capacidade de manter sua posição entre seus concorrentes diretos no mercado } \\
\text { local na posição atual. }\end{array}$ \\
\hline & & $\begin{array}{l}\text { Nosso hospital tem a capacidade de prever sua participação de mercado, considerando a intensidade da } \\
\text { concorrência. }\end{array}$ \\
\hline & & $\begin{array}{l}\text { Nosso hospital tem uma base estratégica para a competição (concorrência em: preço, diferenciação de } \\
\text { serviço, tempo, qualidade). }\end{array}$ \\
\hline & \multirow[t]{3}{*}{ Rapidez } & Nosso hospital pode alterar rapidamente a quantidade de nossos serviços. \\
\hline & & Nosso hospital pode lançar novos serviços no mercado. \\
\hline & & Nosso hospital pode descobrir rapidamente mudanças nas preferências e doenças do cliente. \\
\hline & \multirow[t]{6}{*}{ Flexibilidade } & Nosso hospital tem a capacidade de operar eficientemente em diferentes níveis de atendimento. \\
\hline & & Nosso hospital pode oferecer diferentes tipos de serviços sem grandes mudanças. \\
\hline & & Trabalhadores em nosso hospital podem realizar diferentes tipos de operações de forma eficaz. \\
\hline & & Trabalhadores em nosso hospital podem realizar uma ampla gama de tarefas de atendimento de forma eficaz. \\
\hline & & Trabalhadores em nosso hospital podem operar vários tipos de equipamentos. \\
\hline & & Trabalhadores em nosso hospital podem ser transferidos facilmente entre unidades organizacionais. \\
\hline \multirow{12}{*}{ 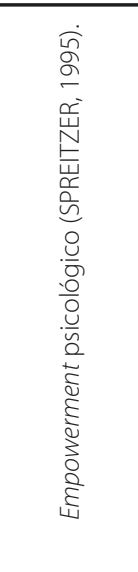 } & \multirow{3}{*}{ Significado } & O trabalho que faço é muito importante para mim \\
\hline & & As atividades diárias da minha profissão são muito significativas para mim \\
\hline & & O trabalho que faço é muito significativo para mim \\
\hline & \multirow[t]{3}{*}{ Competência } & Estou confiante em minhas capacidades no desempenho de minhas funções \\
\hline & & Estou seguro das minhas capacidades no desempenho das minhas funções \\
\hline & & Eu domino as habilidades necessárias das minhas funções \\
\hline & \multirow[t]{3}{*}{ Autodeterminação } & Eu tenho autonomia significativa para determinar como faço meu trabalho \\
\hline & & Eu posso decidir como fazer o meu trabalho \\
\hline & & Eu tenho a liberdade em definir como faço o meu trabalho \\
\hline & \multirow[t]{3}{*}{ Impacto } & Meu trabalho tem grande impacto no meu departamento, área ou unidade \\
\hline & & Eu tenho controle sobre o que acontece no meu departamento, área ou unidade \\
\hline & & Eu tenho influência significativa sobre o que acontece no meu departamento, área ou unidade \\
\hline \multirow{9}{*}{ 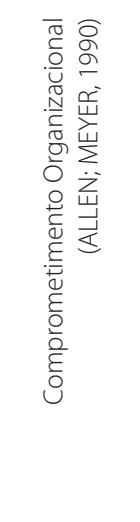 } & \multirow{9}{*}{$\begin{array}{l}\text { Comprometimento } \\
\text { Organizacional }\end{array}$} & Tenho muito orgulho de poder dizer as pessoas onde trabalho \\
\hline & & Não estou disposto a me expor apenas para ajudar o hospital \\
\hline & & $\begin{array}{l}\text { Mesmo que o hospital não estivesse indo muito bem financeiramente, estaria relutante em mudar para } \\
\text { outro hospital }\end{array}$ \\
\hline & & Estou seguro das minhas capacidades no desempenho das minhas funções \\
\hline & & Sinto que sou parte deste hospital \\
\hline & & $\begin{array}{l}\text { Nas minhas funções, gosto de sentir que estou fazendo algum esforço, não apenas para mim, mas } \\
\text { também para o hospital }\end{array}$ \\
\hline & & $\begin{array}{l}\text { Se o meu salário fosse inferior ao do meu colega que desempenha a mesma função que eu, não me faria } \\
\text { mudar de hospital }\end{array}$ \\
\hline & & Não recomendaria a um amigo próximo a se candidatar a uma vaga de emprego neste hospital \\
\hline & & Saber que meu trabalho contribui para o melhor desempenho do hospital me agrada. \\
\hline
\end{tabular}

Fonte: Dados da Pesquisa. 
por meio de escala Likert de 5 pontos. O primeiro ponto da escala estava relacionado ao quanto o indivíduo discordava com as assertivas e o último ao quanto concordava na totalidade. Apresenta-se a seguir os construtos da pesquisa e suas respetivas assertivas.

Para a análise dos dados utilizou-se a técnica de Modelagem de Equações Estruturais (PLS), estimada a partir dos mínimos quadrados parciais (Partial Least Square). Conforme elucidam Hair Jr. et al. (2016), esta técnica busca examinar as inter-relações entre os construtos na qual usa de forma combinada análise fatorial e regressão múltipla. Ressalta-se que nesta pesquisa seguiu-se as recomendações de Hair Jr. et al. (2016), para testar os dados e a mediação da variável empowerment psicológico.

\section{ANÁLISE E DISCUSSÃO DOS RESULTADOS}

\subsection{Perfil dos Respondentes}

Os respondentes desta pesquisa apresentaram um perfil diversificado os quais são apresentados na Tabela 2.

Pode-se observar de acordo com o perfil dos respondentes, que 66\% são do gênero feminino e 34
$\%$ do gênero masculino, e que a grande maioria tem mais de 22 anos de idade na data da coleta dos dados. Quanto ao grau de formação, a maioria (66\%) possui curso superior concluído, sendo (52\%) graduação e (14\%) pós-graduação. Quanto ao setor predominou o administrativo, com $69 \%$ dos respondentes. Cabe salientar ainda que dos respondentes $13 \%$ fazem parte do corpo médico e $18 \%$ do corpo de enfermeiros.

\subsection{Modelo de Mensuração}

Para testar o modelo de mensuração e adequação dos construtos utilizou-se das validades discriminante e convergente, confiabilidade composta e alfa de cronbach (HAIR Jr. et al., 2016).

$\mathrm{O}$ instrumento de pesquisa apresentava inicialmente 40 questões distribuídas entre os construtos, dessas, na variável agilidade organizacional foram excluídas dez questões (COMP_1 a COMP_4; RESP_1; RESP_5; FLEX_1; FLEX_2; FLEX_5; FLEX_6), três questões no empowerment psicológico (EP_09; EP_10; EP_11), e cinco questões referente ao comprometimento organizacional $\left(\mathrm{CO} \_1 ; \mathrm{CO} \_2 ; \mathrm{CO} \_3\right.$ : $\mathrm{CO} \_6$; CO_7). A exclusão dessas variáveis encontra respaldo na literatura, dado que as cargas fatoriais estavam abaixo do requerido $(0,50)$, o que pode interferir nas validades (descriminante e convergente) e confiabili-

Tabela 2 Perfil dos Respondentes

\begin{tabular}{|c|c|c|c|c|c|}
\hline Gênero & $\mathbf{N}$ & (\%) & Escolaridade & $\mathbf{N}$ & (\%) \\
\hline Feminino & 57 & $66 \%$ & Pós-graduação & 12 & $14 \%$ \\
\hline \multirow[t]{5}{*}{ Masculino } & 30 & $34 \%$ & Ensino Superior & 45 & $52 \%$ \\
\hline & & & Ensino Superior Incompleto & 15 & $17 \%$ \\
\hline & & & Ensino médio & 11 & $13 \%$ \\
\hline & & & Ensino técnico & 3 & $3 \%$ \\
\hline & & & Ensino fundamental & 1 & $1 \%$ \\
\hline Total & 87 & $100 \%$ & Total & 87 & $100 \%$ \\
\hline Setor & $\mathbf{N}$ & (\%) & Idade & $\mathbf{N}$ & (\%) \\
\hline Administrativo & 60 & $69 \%$ & De 18 a 21 anos & 9 & $10 \%$ \\
\hline Corpo Médico & 11 & $13 \%$ & De 22 a 30 anos & 40 & $46 \%$ \\
\hline Corpo da Enfermagem & 16 & $18 \%$ & Acima de 30 anos & 38 & $44 \%$ \\
\hline Total & 87 & $100 \%$ & Total & 87 & $100 \%$ \\
\hline
\end{tabular}

Fonte: Dados da Pesquisa. 
Tabela 3 Validade Discriminante - Fornell e Larcker

\begin{tabular}{l|c|c|c}
\multicolumn{1}{c|}{ Variáveis } & $\begin{array}{c}\text { Agilidade } \\
\text { Organizacional }\end{array}$ & $\begin{array}{c}\text { Comprometimento } \\
\text { Organizacional }\end{array}$ & $\begin{array}{c}\text { Empowerment } \\
\text { psicológico }\end{array}$ \\
\hline Agilidade Organizacional (AO) & $\mathbf{0 , 7 1 4}$ & & \\
\hline Comprometimento Organizacional (CO) & 0,365 & $\mathbf{0 , 7 3 1}$ & \\
\hline Empowerment psicológico (EP) & 0,300 & 0,720 & $\mathbf{0 , 7 2 6}$ \\
\hline
\end{tabular}

Fonte: Dados da Pesquisa.

dade do modelo, além de que em alguns momentos os indicadores dividiam cargas.

O modelo final foi testado com 18 indicadores e resultou nos seguintes coeficientes para a validade discriminante, conforme matriz de Fornell e Larcker (1981).

Os índices da matriz Fornell e Larcker são calculados a partir da raiz quadrada da variância média extraída (average variance extracted-AVE) das variáveis latentes, desse modo quando forem maiores que os valores absolutos das correlações de outras variáveis, pode-se confirmar a validade. Destaca-se que os construtos se mostraram procedentes nos modelos, isto porque são distintos e se adequam a critérios apresentados na literatura.

Após a análise das cargas fatoriais observou-se a validade convergente pelo coeficiente da AVE, o alfa de Cronbach e a confiabilidade composta. A partir do momento que a AVE for maior que 0,5 , pode-se confirmar a validade do modelo. Em relação a confiabilidade, Hair Jr. et al. (2016) apontam que o modelo é confiável se o alfa de Cronbach, for superior a 0,7. A Tabela 4 apresenta os resultados do modelo de mensuração.

Mediante os resultados da AVE, pode-se observar que o modelo é válido dado que os índices foram acima de 0,50.Do mesmo modo que a AVE, a partir do alfa de Cronbach verifica-se que o modelo é confiável, dado que os valores estão acima de 0,7. A confiabilidade composta, no entanto, apresentou melhores índices, sendo que todos estão entre 0,82 , a 0,91 , o que evidencia alta confiabilidade dos construtos

\subsection{Modelo Estrutural}

Para avaliar a validade do modelo estrutural são analisados os seguintes critérios: (i) o tamanho e significância dos coeficientes de caminho, (ii) coeficientes de determinação de Pearson $\left(\mathrm{R}^{2}\right)$, (iii) tamanhos do efeito $\left(\mathrm{F}^{2}\right)$ e, (iv) a $\left(\mathrm{Q}^{2}\right)$ Relevância Preditiva (HAIR Jr. et al., 2016). Destaca-se que os dois primeiros itens são obtidos por meio da técnica de Bootstrapping e os itens iii e iv são na plataforma blindfolding. Foram testados 5.000 subamostras e 5.000 interações em um intervalo de confiança de Bias-Corrected and accelerated a nível de significância de 5\% e unicaudal para examinar as hipóteses da pesquisa, confirmar a adequação do modelo de mensuração e testar a significância das relações entre as variáveis (HAIR Jr. et al., 2016). Pode-se observar na Tabela 5, a apresentação dos caminhos e dos valores do $\mathrm{R}^{2}$ e do $\mathrm{Q}^{2}$.

Ao se analisar o resultado de determinação de Pearson $\left(\mathrm{R}^{2}\right)$, verificou-se que para o coeficiente do empowerment psicológico é de $9 \%$. Considera-se médio este valor logo, pode-se afirmar que a agilidade organizacional tem um poder explicativo de quase $10 \%$. Contudo, ao se observar o resultado para o

Tabela 4 Resultados do modelo de mensuração

\begin{tabular}{l|c|c|c}
\multicolumn{1}{c|}{ Variáveis } & (AVE) & Alpha Cronbach & $\begin{array}{c}\text { Confiabilidade com- } \\
\text { posta }\end{array}$ \\
\hline Agilidade Organizacional & 0,510 & 0,760 & 0,838 \\
\hline Comprometimento Organizacional & 0,534 & 0,708 & 0,820 \\
\hline Empowerment psicológico & 0,528 & 0,887 & 0,909 \\
\hline
\end{tabular}

Fonte: Dados da Pesquisa. 
Tabela 5 Apresentação dos resultados.

\begin{tabular}{l|c|c|c}
\multicolumn{1}{c|}{ Relação entre os construtos } & B & p-valor & Hipótese \\
\hline Agilidade organizacional $\rightarrow$ Comprometimento organizacional & 0,164 & $0,040^{* *}$ & $\mathrm{H} 1$ \\
\hline Empowerment psicológico $\rightarrow$ Comprometimento Organizacional & 0,662 & $0,000^{* * *}$ & $\mathrm{H} 2$ \\
\hline Agilidade organizacional $\rightarrow$ Empowerment psicológico $\rightarrow$ Comprometimento organizacional & 0,210 & $0,002^{* * *}$ & $\mathrm{H3}$ \\
\hline
\end{tabular}

Nota $1 \mathrm{~N}=87 ;{ }^{*} \mathrm{p}<0.10 ;{ }^{* *} \mathrm{p}<0.05 ;{ }^{* * *} \mathrm{p}<0.01$

Nota $2 R^{2}=$ Empowerment psicológico (0,09); Comprometimento organizacional $(0,542)$; $\mathrm{Q}^{2}=$ Empowerment psicológico $(0,036)$; Comprometimento organizacional $(0,243)$.

Fonte: Dados da Pesquisa.

comprometimento organizacional constatou-se um $\mathrm{R}^{2}$ de $54,20 \%$, o qual é considerado um grande efeito pois a agilidade organizacional em conjunto com o empowerment psicológico explicam mais de $50 \%$ do modelo, e evidenciam sua validade preditiva. Quanto ao $Q^{2}$, Hair Jr. et al. (2016) ressaltam que este indicador avalia a acurácia do modelo ajustado, e para tanto, quanto maior que (0) melhor, logo para o empowerment psicológico o $\mathrm{Q}^{2}$ foi de $3,3 \%$ e para o comprometimento organizacional $24,3 \%$, o que evidencia que o modelo é condizente e ajustado e com relevância preditiva.

Quanto às hipóteses, evidencia-se a partir da Tabela 5 que os efeitos diretos da agilidade organizacional e o comprometimento organizacional são positivos e significantes a nível de $5 \%(\beta: 0,164$, p-valor $<0,050)$, logo confirma-se a $\mathrm{H}_{1}$. Esses achados são convergentes com Farahani e Salimi (2015) que verificaram a relação positiva e significativa entre agilidade organizacional e o comprometimento organizacional. Essa relação foi confirmada, pois os funcionários perceberam que o hospital em estudo consegue adaptar-se às estratégias, processos e recursos conforme as mudanças no ambiente. Tal percepção gerou neles sentimento de segurança durante o desempenho de suas funções e de pertencimento à organização (ALLEN; MEYER, 1990; BAIRD; TUNG; YU, 2019).

Quanto à relação entre o empowerment psicológico e o comprometimento organizacional, confirma-se a $\mathrm{H}_{2}$, pois constata-se um coeficiente $\beta$ de 0,670 e o p-valor $<0,001$, que evidencia um efeito positivo e significativo do empowerment psicológico no comprometimento organizacional. Pode-se inferir que as dimensões do significado, competência, autodeterminação e impacto dos funcionários apre- sentam uma forte ligação com seu comprometimento. Isso se dá em razão de que o comprometimento se refere à sentimentos de apego à organização (KIM et al., 2002), logo, quanto mais o indivíduo se sentir psicologicamente empoderado maior será seu comprometimento. Este resultado vai ao encontro dos obtidos no estudos de Kouklanen et al. (2003), ao observarem que o empowerment psicológico é positivamente relacionado com o comprometimento organizacional, além de que em um ambiente hospitalar os funcionários se sentem mais empoderados, e consequentemente se comprometem mais com a organização.

$\mathrm{Na} \mathrm{H}_{3}$, conjecturou-se a mediação do empowerment psicológico. Bido e Silva (2019) apontam que a mediação parcial ocorre quando o efeito direto é significativo ao mesmo tempo que o efeito indireto. Logo, foi possível confirmar parcialmente $\mathrm{H}_{3}$, tendo em vista que na relação indireta o coeficiente $\beta$ foi de 0,201 e o p-valor $<0,010$, do mesmo modo que a relação direta foi significante, conforme a primeira hipótese. Desse modo foi possível observar uma relação positiva e significante entre a agilidade organizacional e o comprometimento organizacional mediada parcialmente pelo empowerment psicológico. Apresenta-se a síntese dos resultados na figura2.

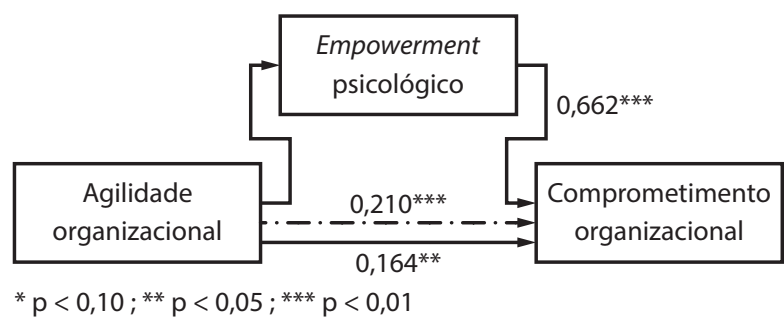

Figura 2 Resumo dos resultados 
Os resultados permitem inferir que os efeitos indiretos do empowerment psicológico reforça a relação entre a variável dependente e independente. denota-se que a agilidade organizacional levará o funcionário a um maior comprometimento, se este, influenciar no seu empowerment psicológico. Esses achados são convergentes com Avolio et al. (2004), dado que constaram que o empowerment psicológico enquanto variável mediadora afeta positivamente o comprometimento organizacional de médicos e enfermeiros de um hospital na Cingapura.

Os funcionários perceberam o significado do trabalho porque possuem autonomia para desempenharem suas atividades com maior liberdade (SPREITZER, 1995). Isso porque no hospital investigado, o ambiente de trabalho é saudável e em contantes mudanças de reformulação de políticas em vista de maior eficiência na prestação dos serviços (THANACOODY; NEWMAN; FUCHS, 2014), panoramas que encorajaram os funcionários. Portanto, em resposta as mudanças no ambiente, os funcionários aumentaram o comprometimento com o hospital (BAIRD; TUNG; YU, 2019).

De modo geral, os achados sustentam as evidências de que a agilidade organizacional influencia positivamente no comprometimento organizacional dos funcionários, e que recursos cognitivos (empowerment psicológico) também são fortes influenciadores tanto para o aumento do comprometimento organizacional, quanto para reforçar a relação entre a agilidade organizacional e o comprometimento dos funcionários.

Esses fatos, despertam atenção dos gestores hospitalares, pois ações proativas em direção à criação de um ambiente saudável são pertinentes para a congruência entre os objetivos dos funcionários e dos hospitais. A pesquisa sugere que as organizações hospitalares incorporem práticas inerentes a agilidade e empowerment psicológico, o que pode implicar em um melhor desempenho organizacional. Esses achados são contribuições que geram importantes avanços na literatura.

\section{CONSIDERAÇÕES FINAIS}

Este estudo teve por objetivo examinar o efeito da agilidade organizacional no comprometimento organizacional mediado pelo empowerment psicológico. A pesquisa foi desenvolvida a partir da percepção dos funcionários de um Hospital com atendimento de excelência em emergências cardiológicas de Florianópolis/SC. Os resultados trazem contribuições importantes para o processo de tomada de decisão dos gestores, em uma estrutura organizacional complexa como são as organizações hospitalares.

Os achados evidenciam que há uma relação direta e positiva entre a agilidade organizacional e o comprometimento organizacional com p-valor $<0,050$, logo confirma-se a $\mathrm{H}_{1}$. No tocante à $\mathrm{H}_{2}$, testou-se a relação entre o empowerment psicológico e o comprometimento organizacional, e confirmou-se a hipótese a um nível de significância de $1 \%$. E quanto $\mathrm{a}_{3}$ observou-se que o empowerment psicológico medeia parcialmente a relação ente a agilidade organizacional e o comprometimento organizacional, a um nível de significância $1 \%$.

A partir do constatado nos resultados, depreende-se que os funcionários do hospital em estudo percebem que a sua organização é ágil, tendo em vista que adotam políticas, as quais permitem adaptar-se às mudanças no ambiente organizacional. Essa percepção aumenta o empoderamento dos funcionários, e consequentemente em seu sentimento de apego com a organização, ou seja, aumentam o comprometimento. Denota-se que, quando os funcionários percebem que sua organização é ágil, vislumbram sua permanência na organização, diminuindo a rotatividade do pessoalo que pressupõe a responsabilidade corporativa da organização, não só com os funcionários, mas também com seus pacientes.

A percepção de que a organização é socialmente responsável, ocasiona empowerment psicológico nos seus funcionários. Sendo as dimensões do empoderamento destacadas em significado, competência, autodeterminação e impacto, evidenciam a consistência desse argumento. Isto porque enquanto variável mediadora produz um efeito indireto entre a agilidade organizacional, destacadas nas dimensões (responsabilidade e flexibilidade) e o comprometimento organizacional. Logo, pela desagregação dos 
construtos pode-se verificar que a responsabilidade e a flexibilidade da organização, leva o funcionário a um maior empoderamento, e consequentemente o comprometimento com a organização. Bahrami et al. (2016) confirmam esses achados, pois afirmam que o comprometimento dos funcionários com a organização é uma questão crucial para o sistema de saúde. Infere-se a partir dessas constatações que organizações similares precisam ter um olhar sobre a responsabilidade e flexibilidade, com a finalidade de melhorar o comprometimento dos seus funcionários, a qualidade de seus serviços e a busca por vantagem competitiva no ambiente organizacional.

O estudo mostra que as organizações devem prestar maior atenção em suas estratégias, processos e recursos. Fatores que melhoram o entendimento sobre a agilidade dos hospitais e permitem que estes alcancem seus objetivos (ALMAHAMID; AWWAD; MCADMAS, 2010). Nesse contexto, o estudo avança na literatura dado que organizações com estruturas complexas como os hospitais estão subjacentes a variáveis contingenciais. E quando analisadas em contextos diferentes possibilitam reflexões sobre como devem se comportar no mercado para alcançar a competitividade. Outro ponto, é o fato de adotar o empowerment psicológico como variável mediadora, observa-se que esta tem um efeito indispensável, visto que a ligação direta apresenta um valor $\mathrm{p}<0,050$, entretanto quando incluído a variável mediadora, o efeito indireto foi significante ao nível de $1 \%$. Isto evidencia a importância do empowerment psicológico dos funcionários para o alcance do comprometimento.

No âmbito prático, este estudo contribui para que gestores de organizações hospitalares, possam compreender melhor a agilidade de suas organizações e propor formas para melhorar suas políticas, estratégias e processos, visando o melhor desempenho a partir do aumento do empoderamento e comprometimento dos seus funcionários. Essa observação vai ao encontro de Vandenberghe e Tremplay (2008), por argumentarem que o comprometimento organizacional é essencial para o alcance de um melhor desempenho. Ademais, a agilidade ajuda a organização a diminuir o índice de desistência do emprego, e por sua vez permite maior engajamento e comprometimento (MATHIEU; ZAJAC, 1990).
Como toda pesquisa científica esse estudo apresenta limitações. A pesquisa investigou funcionários de diferentes funções na organização, fator que pode ser entendido como uma limitação. As percepções dos funcionários podem variar de acordo o cargo ocupa. Logo ao mesmo tempo que é vantajoso olhares distintos, também pode ter limtado a análise. Outro ponto é que a pesquisa optou por um modelo com variáveis multidimensionais, outras pesquisas podem adotar variáveis unidimensionais e em um contexto onde os indivíduos sejam do mesmo grupo. Futuras pesquisas poderiam também se ater em outras variáveis como, por exemplo, o efeito do turnover como variável moderadora da relação entre a agilidade organizacional e o comprometimento.

\section{REFERÊNCIAS}

ALAVI, Somaieh et al. Organic structure and organisational learning as the main antecedents of workforce agility. International Journal of Production Research, v. 52, n. 21, p. 6273-6295, 2014.

ALLEN, Natalie J.; MEYER, John P. The measurement and antecedents of affective, continuance and normative commitment to the organization. Journal of occupational psychology, v. 63, n. 1, p. 1-18, 1990.

ALMAHAMID, Soud; AWWAD, Abdulkareem; MCADAMS, Arthur C. Effects of organizational agility and knowledge sharing on competitive advantage: an empirical study in Jordan. International Journal of Management, v. 27, n. 3, p. 387, 2010.

AVOLIO, Bruce J. et al. Transformational leadership and organizational commitment: Mediating role of psychological empowerment and moderating role of structural distance. Journal of Organizational Behavior: The International Journal of Industrial, Occupational and Organizational Psychology and Behavior, v. 25, n. 8, p. 951-968, 2004. 
BAHRAMI, Mohammad Amin et al. The mediating role of organizational learning in the relationship of organizational intelligence and organizational agility. Osong public health and research perspectives, v. 7, n. 3, p. 190-196, 2016.

BAIRD, Kevin M.; TUNG, Amy; YU, Yanjie. Employee organizational commitment and hospital performance. Health care management review, $v$. 44, n. 3, p. 206-215, 2019.

BIDO, Souza Diógenes; SILVA, Dirceu. SmartPLS 3: especificação, estimação, avaliação e relato. Administração: Ensino e Pesquisa, v. 20, n. 2, p. 1-31, 2019.

BIN JOMAH, Nouf. Psychological empowerment on organizational commitment as perceived by Saudi academics. World Journal of Education, v. 7, n. 1, p. 83-92, 2017.

Brislin, R. W. Back-translation for cross-cultural research. Journal of Cross-Cultural Psychology, 1(3), 185-216, 1970.

BROWN, Steve; BESSANT, John. The manufacturing strategy-capabilities links in mass customization and agile manufacturing-an exploratory study. International Journal of Operations \& Production Management, v. 23, n. 7, p. 707-730, 2003.

CHARBONNIER-VOIRIN, Audrey. The development and partial testing of the psychometric properties of a measurement scale of organizational agility. Management, v. 14, n. 2, p. 120, 2011.

CONGER, Jay A.; KANUNGO, Rabindra N. The empowerment process: Integrating theory and practice. Academy of Management Review, v. 13, n. 3, p. 471-482, 1988.

CONWAY, Edel; MONKS, Kathy. Unravelling the complexities of high commitment: an employee-level analysis. Human Resource Management Journal, v. 19, n. 2, p. 140-158, 2009.
FARAHANI, Alireza H.; SALIMI, Freidoon. The Study of the Relationship between Employees' Empowerment and Organizational Agility: A Case Study in Azarab Industrial Company. European Online Journal of Natural and Social Sciences: Proceedings, v. 4, n. 1 (s), p. 067-1075, 2015.

FAUL, Franz et al. Statistical power analyses using $G^{\star}$ Power 3.1: Tests for correlation and regression analyses. Behavior Research Methods, v. 41, n. 4, p. 1149-1160, 2009.

FORNELL, Claes; LARCKER, David F. Structural equation models with unobservable variables and measurement error: Algebra and statistics. 1981.

HAIR JR, Joseph F. et al. A primer on partial least squares structural equation modeling (PLS-SEM). Sage publications, 2016.

KIM, Beom Cheol et al. Motivational effects of empowerment on employees' organizational commitment: a mediating role of management trustworthiness. Cornell Hospitality Quarterly, v. 53, n. 1, p. 10-19, 2012.

MATHIEU, John E.; ZAJAC, Dennis M. A review and meta-analysis of the antecedents, correlates, and consequences of organizational commitment. Psychological bulletin, v. 108, n. 2, p. 171, 1990.

MEHDIBEIGI, Najme; DEHGHANI, Masoud; MOHAMMAD YAGHOUBI, Nour. Customer knowledge management and organization's effectiveness: explaining the mediator role of organizational agility. Procedia-Social and Behavioral Sciences, v. 230, p. 94-103, 2016.

MOWDAY, Richard T.; PORTER, Lyman W.; STEERS, Richard M. Employee-organization linkages: The psychology of commitment, absenteeism, and turnover. Academic press, 2013.

NAFEI, Wageeh A. Organizational agility: The key to organizational success. International Journal of Business and Management, v. 11, n. 5, p. 296-309, 2016. 
PORKIANI, Masood; HEJINIPOOR, Mohsen. Studying the relationship between organizational intelligence and organizational agility in supreme audit court. European Online Journal of Natural and Social Sciences: Proceedings, v. 2, n. 3 ,p. 10521060, 2013.

SAMBAMURTHY, Vallabh; BHARADWAJ, Anandhi; GROVER, Varun. Shaping agility through digital options: Reconceptualizing the role of information technology in contemporary firms. MIS quarterly, p. 237-263, 2003.

SHARIFI, Hossein; ZHANG, Zhengwen. A methodology for achieving agility in manufacturing organisations: An introduction. International Journal of Production Economics, v. 62, n. 1-2, p. 7-22, 1999.

SHAPIRA-LISHCHINSKY, Orly; TSEMACH, Sigalit. Psychological empowerment as a mediator between teachers' perceptions of authentic leadership and their withdrawal and citizenship behaviors. Educational Administration Quarterly, v. 50, n. 4, p. 675-712, 2014.

SPREITZER, Gretchen M. Psychological empowerment in the workplace: Dimensions, measurement, and validation. Academy of Management Journal, v. 38, n. 5, p. 1442-1465, 1995.

STEERS, Richard M. Antecedents and outcomes of organizational commitment. Administrative Science Quarterly, p. 46-56, 1977.

THANACOODY, P. R.; NEWMAN, A.; FUCHS, Sebastian. Affective commitment and turnover intentions among healthcare professionals: The role of emotional exhaustion and disengagement. The International Journal of Human Resource Management, v. 25, n. 13, p. 1841-1857, 2014.

THOMAS, Kenneth W.; VELTHOUSE, Betty A. Cognitive elements of empowerment: An "interpretive" model of intrinsic task motivation. Academy of Management Review, v. 15, n. 4, p. 666-681, 1990.
VANDENBERGHE, Christian; TREMBLAY, Michel. The role of pay satisfaction and organizational commitment in turnover intentions: A two-sample study. Journal of Business and Psychology, v. 22, n. 3, p. 275-286, 2008.

WANG, Yingyan. Examining organizational citizenship behavior of Japanese employees: A multidimensional analysis of the relationship to organizational commitment. The International Journal of Human Resource Management, v. 26, n. 4, p. 425-444, 2015.

YUSUF, Yahaya Y.; SARHADI, Mansoor; GUNASEKARAN, Angappa. Agile manufacturing: The drivers, concepts and attributes. International Journal of Production Economics, v. 62, n. 1-2, p. 33-43, 1999.

ZHANG, Z.; SHARIFI, H. Uma metodologia para alcançar agilidade nas organizações de manufatura. Revista Internacional de Operações e Gestão de Produção, v.20, n.4, p. 496-513, 2000. 